\title{
命HAD
}

DOI: http://doi.org/10.22585/hospdomic.v4i3.106

\section{Hospitalización domiciliaria en un área rural, ¿̇una alternativa a la hospitalización tradicional?}

\author{
Home care services provided by hospital in a rural area, \\ an alternative to traditional hospitalization?
}

Paula Hernández Martínez', Olalla Fernández Revilla', Daniel Ahumada González', $M^{a}$ del Pilar Ruiz Seco', Laura Calderón Mediavilla', Azucena Roldán Luis ${ }^{2}$

1. Hospital Sierrallana y Tres Mares. Torrelavega, España.

Correspondencia/Correspondence

Paula Hernández Martínez

paulaherma@gmail.com

Recibido/Received

16.04.2020

Aceptado/Accepted

24.04.2020
Conflicto de Intereses/Competing interest Los autores afirmar no presentar ningún conflicto de interés.

Financiación/Fundings

Este trabajo no ha recibido ningún tipo de financiación. 


\section{RESUMEN}

Introducción: El servicio de hospitalización domiciliaria en el área rural es un servicio poco conocido en el resto del ámbito sanitario. Al mismo tiempo existe poca literatura al respecto por lo que con este estudio queremos estudiar las características clínicas, la estancia media y la tasa de reingresos de los pacientes que ingresan en hospitalización domiciliaria en comparación a los pacientes que ingresan en planta de medicina interna pertenecientes a un hospital comarcal de un área rural (Hospital de TresMares).

Método: Estudio descriptivo comparativo retrospectivo que incluye un total de 200 pacientes (100 ingresados en hospitalización domiciliaria y 100 ingresados en planta) del hospital de TresMares

Resultados: La complejidad de los pacientes que ingresan en planta es superior a los que ingresan en hospitalización domiciliaria requiriendo al mismo tiempo de mayor uso de recursos. La eficacia, entendida como tasa de reingreso, en hospitalización a domicilio es similar a la de los pacientes de la planta. Se demuestra que la hospitalización domiciliaria puede ser una alternativa a la hospitalización tradicional en un área rural.

Conclusiones: Se demuestra que la hospitalización domiciliaria puede ser una alternativa a la hospitalización tradicional en un área rural.

Palabras clave: Servicios de Atención de Salud a Domicilio; Servicios de Atención a Domicilio Provisto por Hospital; Medio Rural; Tiempo de Internación.

\section{ABSTRACT}

Introduction: Hospital home care in a rural area is a hospital care little known in health field. At the same time there is little literature so we want to study the clinical characteristics, the average stay and the readmission rate of patients admitted to home hospitalization compared to patients admitted to the internal medicine hospitalization to a regional hospital in a rural area (Hospital de TresMares).

Method: retrospective comparative descriptive study includes 200 patients (100 patients admitted to home hospitalization and 100 patients admitted to the hospital)

Results: the complexity of the patients admitted to the hospital is greater than those admitted to home hospitalization, while requiring greater use of resources. The efficacy, understood as re admission rate, in hospitalization at home is similar that the patients who are admitted in the hospital.

Conclusions: home hospitalization can be an alternative to tradicional hospitalization in a rural area.

Keywords: Home Care Services; Home Care Services, Hospital-Based; Rural Areas; Length of Stay. 


\section{INTRODUCCIÓN}

La hospitalización domiciliaria nació en 1947 en el hospital universitario Guido Montefiore de Nueva York y no es hasta 1981 que surge la primera unidad en España, en el antiguo Hospital Provincial de Madrid. Desde entonces este sistema asistencial ha ido creciendo por el territorio español inicialmente con el objetivo de evitar ingresos en el hospital y realizar altas precoces desde las plantas de hospitalización permitiendo descongestionar los hospitales, para posteriormente consolidarse como la primera opción asistencial para algunos procesos $(1,2)$.

En la última revisión realizada por la Cochrane en 2017 en el que se compara el modelo asistencial de ingreso hospitalario tradicional versus ingreso domiciliario no han evidenciado diferencias en cuanto a la mortalidad (nivel de evidencia moderada), ni de reingreso (nivel de evidencia baja), ni tampoco de ahorro de costes, aunque sí parece disminuir los días de ingreso y aumentar la satisfacción de los pacientes (nivel de evidencia baja) (3). Ante esta incertidumbre decidimos estudiar cuál es nuestra realidad, teniendo en cuenta la escasa bibliografía comparativa entre dichos modelos asistenciales a nivel nacional y la inexistente si matizamos que somos un servicio de hospitalización domiciliaria que desarrolla su actividad en medio rural.

El hospital de TresMares, espacio sanitario donde se desarrolla nuestro estudio, está ubicado en la localidad de Reinosa, en Cantabria, y presta servicio a la comarca de Campoo- Los Valles. Dicha comarca tiene una extensión de unos 1012 km2, con una población aproximada de 20520 habitantes. El hospital TresMares comenzó su actividad en el año 2010 y está dotado de un área de hospitalización que incluye 20 habitaciones dobles, un área de Urgencias, una sala de Hospital de Día, dos quirófanos para cirugía mayor ambulatoria, además de un área de radiodiagnóstico y una zona de consultas. El 1 de noviembre de 2018 se añadió a su cartera de servicios Hospitalización domiciliaria con un total de 12 camas y una isócrona de 20 minutos, en la que se excluyen pacientes que viven en residencias.

\section{MÉTODOS}

Estudio descriptivo y comparativo de los pacientes ingresados en hospitalización domiciliaria de un hospital comarcal de un área rural y los pacientes ingresados en planta de medicina interna del mismo hospital.

Se recogieron los datos de los 100 primeros pacientes que ingresaron en hospitalización domiciliaria desde el 1 de noviembre de 2018 excluyendo aquellos pacientes que tuvieron estancia en diferentes servicios durante ese ingreso; es decir, aquellos pacientes que inicialmente ingresaron en planta de medicina interna o fueron trasladados desde otro hospital para posteriormente completar tratamiento en el domicilio, y al mismo tiempo aquellos pacientes que iniciaron tratamiento en su domicilio y por la evolución requirieron de ingreso en planta o traslado.

Para seleccionar los pacientes ingresados en planta buscamos en los listados de admisión aquellos que ingresaban con la misma fecha que los pacientes de domiciliaria (en el caso de que hubiese varios pacientes se escogía aquel que se acercase más a la hora del ingreso del paciente de hospitalización domiciliaria).

Finalmente se estudiaron un total de 92 pacientes en el grupo de hospitalización domiciliaria y 97 en hospitalización en planta ya que hubo pacientes de los que no se pudieron extraer todos los datos para el estudio o no cumplían los criterios de selección. 


\section{RESULTADOS}

Los parámetros epidemiológicos y clínicos que se estudiaron se resumen en la tabla número 1.

Tabla 1. Parámetros epidemiológicos y clínicos

\begin{tabular}{|c|c|c|c|}
\hline & $\begin{array}{l}\text { Hospitalización domiciliaria } \\
(n=92)\end{array}$ & $\begin{array}{l}\text { Hospitalización en planta } \\
(n=97)\end{array}$ & p-valor \\
\hline Sexo & 33 mujeres $(35,8 \%)$ & 47 mujeres $(48,4 \%)$ & \\
\hline Edad (años) & $79,49 \pm 12,60$ & $84,31 \pm 10,36$ & 0,004 \\
\hline Crónicos pluripatológicos & $41,3 \%(38)$ & $63,9 \%(62)$ & 0,001 \\
\hline Escala de Barthel & $77,28 \pm 32,87$ & $47,89 \pm 39,03$ & $<0,0001$ \\
\hline Escala de Charlson & $5,87 \pm 2,59$ & $6,58 \pm 2,34$ & 0,05 \\
\hline Días de estancia media & $10,85 \pm 5,76$ & $4,86 \pm 37,88$ & 0,13 \\
\hline Institucionalizados & $0 \%$ & $36 \%(35)$ & $<0,0001$ \\
\hline $\begin{array}{l}\text { Motivo de ingreso más } \\
\text { frecuente }\end{array}$ & $\begin{array}{l}\text { Infec resp / neumonía 44,5\% } \\
\text { (41) }\end{array}$ & $\begin{array}{l}\text { Infec resp/ neumonía 56,7\% } \\
\text { (55) }\end{array}$ & \\
\hline $\begin{array}{l}2^{\circ} \text { motivo de ingreso más } \\
\text { frecuente }\end{array}$ & Infección de orina 19,5\% (18) & $\begin{array}{l}\text { Insuficiencia cardiaca } 16,4 \% \\
\text { (16) }\end{array}$ & 0,7 \\
\hline Antibioterapia E.V. & $69,5 \%(64)$ & $85,5 \%(83)$ & 0,003 \\
\hline Tratamiento nebulizado & $39,1 \%(34)$ & $57,7 \%(56)$ & 0,002 \\
\hline Oxigenoterapia & $39,1 \%(36)$ & $73,1 \%(71)$ & $<0,0001$ \\
\hline Corticoterapia E.V. & $33,6 \%(31)$ & $43,2 \%(42)$ & 0,11 \\
\hline Diuréticos E.V. & $25 \%(23)$ & $46,3 \%(45)$ & 0,0015 \\
\hline $\begin{array}{l}\text { Cambios en su tratamiento } \\
\text { habitual }\end{array}$ & $32,6 \%(30)$ & $45,3 \%(44)$ & 0,04 \\
\hline Antibioterapia al alta & $19,5 \%(18)$ & $34 \%(33)$ & 0,01 \\
\hline Corticoterapia al alta & $30,4 \%(28)$ & $22,6 \%(22)$ & 0,34 \\
\hline
\end{tabular}




\begin{tabular}{|c|c|c|c|}
\hline & $\begin{array}{l}\text { Hospitalización domiciliaria } \\
(n=92)\end{array}$ & $\begin{array}{l}\text { Hospitalización en planta } \\
(n=97)\end{array}$ & p-valor \\
\hline Oxigenoterapia al alta $\left(^{*}\right)$ & $18,4 \%(17)$ & $10,3 \%(10)$ & 0,15 \\
\hline $\begin{array}{l}\text { Urgencias en }<30 \text { días } \\
\text { al alta }\end{array}$ & $18,4 \%(17)$ & $10,3 \%(10)$ & 0,15 \\
\hline $\begin{array}{l}\text { Media de días hasta la } \\
\text { asistencia en urgencias }\end{array}$ & $13,5 \pm 7,3$ & $14,68 \pm 9,7$ & 0,63 \\
\hline $\begin{array}{l}\text { Reingreso en }<30 \text { días } \\
\text { al alta }\end{array}$ & $27,1 \%(25)$ & $20,6 \%(20)$ & 0,41 \\
\hline $\begin{array}{l}\text { Medias de días hasta el } \\
\text { reingreso }\end{array}$ & $13,84 \pm 6,7$ & $14,31 \pm 8,8$ & 0,86 \\
\hline Exitus & $3,2 \%(3)$ & $18,5 \%(18)$ & 0,0009 \\
\hline \multicolumn{4}{|c|}{$\begin{array}{l}\text { E.V. endovenosa } \\
\left({ }^{*}\right) \text { en caso de que el paciente no tuviese previamente este tratamiento }\end{array}$} \\
\hline
\end{tabular}

Más de la mitad de los pacientes que ingresaron tanto en el hospital como en el domicilio fueron varones $(64,2 \%)$. La edad media de los pacientes que ingresaron en el servicio de hospitalización a domicilio fue significativamente inferior (79 años) respecto a los pacientes que ingresaron en hospitalización (84 años) ( $p=0,004)$.

Se analizó el porcentaje de pacientes que cumplían criterios de paciente crónico pluripatológico siguiendo la definición de Ollero. Se alcanzó un 63,9\% en la hospitalización tradicional siendo superior significativamente a los pacientes crónicos pluripatológicos de hospitalización domiciliario ( $\mathrm{p}=$ 0,001), no siendo tampoco desdeñable su prevalencia en dicho grupo $(41,3 \%)$

La complejidad de los pacientes que ingresan en hospitalización en planta es claramente superior a los pacientes de hospitalización domiciliaria $(p<0,0001)$ con un Barthel medio de 47,89 en comparación al Barthel medio de los pacientes de hospitalización domiciliaria de 77,28. La puntuación en la escala de Charlson en hospitalización domiciliaria fue de 5,87 encontrándose al límite de la significación estadística $(p=0,05)$ en comparación con el grupo de hospitalización en planta (6,58 de puntuación media de Charlson).

No encontramos diferencias estadísticamente significativas entre ambos grupos al analizar la estancia media, viendo además que el principal motivo de ingreso en ambas poblaciones fue la infección respiratoria/neumonía, seguido de la infección de orina en el grupo de hospitalización domiciliaria y de insuficiencia cardiaca en hospitalización en planta.

En relación al tratamiento, el uso de la vía endovenosa, los tratamientos nebulizados y el uso de oxigenoterapia fue menor de manera estadísticamente significativa en el grupo de los pacientes ingresados en hospitalización domiciliaria, salvo el uso de la corticoterapia. Aproximadamente un tercio de los pacientes de hospitalización fueron dados de alta con prescripción de antibioterapia, y una quinta parte con corticoterapia vía oral. En cambio, a los pacientes ingresados en hospitalización domiciliaria no llegó a una quinta parte los pacientes con prescripción de antibioterapia al alta, 
con cifras similares al grupo de pacientes ingresados en hospitalización en cuanto al tratamiento corticoideo.

No encontramos diferencias estadísticamente significativas entre ambos grupos cuando comparamos el porcentaje de pacientes que acude a urgencias antes de los 30 días tras el alta, ni en la proporción de aquellos que requieren de reingreso. Tampoco se encontraron diferencias en la media de días que pasaron hasta la nueva asistencia sanitaria.

\section{DISCUSIÓN}

En nuestro trabajo observamos que la complejidad de los pacientes que ingresan en hospitalización es superior a los que ingresan en domiciliaria (con una edad y un índice de Charlson superior y una puntuación en la escala de Barthel inferior en el primer grupo). En ambos grupos se encuentra un porcentaje importante de pacientes crónicos pluripatológicos. Por otra parte, los recursos que requieren el grupo que ingresa en hospitalización domiciliaria es significativamente inferior a los pacientes de la hospitalización en planta (requieren menos antibioterapia endovenosa, tratamiento nebulizado y oxigenoterapia) por lo que se asume que el coste del ingreso es también inferior. El motivo de ingreso principal en ambos grupos es la infección de origen respiratorio y los días de estancia media fueron también similares, sin encontrarse tampoco diferencias en cuanto a la tasa de reingresos. En cuanto a la tasa de exitus, aunque en otras revisiones como a la que se hace referencia en la introducción la tasa de mortalidad es similar, en nuestro estudio la cantidad de pacientes fallecidos es superior en la planta y creemos que pueda estar en relación a la mayor complejidad de los pacientes que ingresan en planta.

\section{BIBLIOGRAFÍA}

1. Gonçalves-Bradley DC, Iliffe S, Doll HA, Broad J, Gladman J, Langhorne P, et al. Early discharge hospital at home. Cochrane Database Syst Rev. 2017;6:CD000356. DOI: 10.1002/14651858. CD000356.pub4; PMID: 28651296

2. Massa Domínguez B. La hospitalización a domicilio en el siglo XXI. Hosp Domic. 2017;1(1):7-9. DOI: 10.22585/hospdomic.v111.8

3. González Ramallo VJ, Valdivieso Martínez B, Ruiz García V. Hospitalización a domicilio. Med Clin (Barc). 2002;118 (17):659-64. DOI: 10.1016/s0025-7753(02)72488-3; PMID: 12028904 